\title{
Welcome to Surfaces-A New Open Access Journal for an Interdisciplinary Scientific Community
}

\author{
Gaetano Granozzi \\ Editor-in-Chief of Surfaces, Department of Chemical Sciences, University of Padova, Via Marzolo 1, \\ 35131 Padova, Italy; gaetano.granozzi@unipd.it; Tel.: +39-049-8275158; Fax: +39-049-8275829
}

Received: 27 March 2018; Accepted: 27 March 2018; Published: 30 March 2018

Surfaces are ubiquitous. Everyday life gives plenty of examples where surfaces and interfaces play a leading role. The first rigorous studies on surfaces date back to the decade of 1960-1970 [1], when Surface Science appeared as an independent discipline: pioneering work on electron diffraction of surfaces gave first structural insights into the 2D world [2-4] and the exploitation of electron inelastic scattering gave rise to chemical analysis of surfaces [5]. Since then, Surface Science developed as an independent discipline, where a limited community of super-specialists built the basis for a deep understanding of the innovative properties induced by surfaces and interfaces. Nowadays, the scenario is completely changed: the concepts of Surface Science are now well-established and its methodological approach is spread all over an interdisciplinary scientific community. The awareness that surfaces are not only a restricted portion of the material but the locus where materials interact with the external world and that many of the materials' functionalities are mostly influenced by the surface properties is now common science heritage. Besides, surface scientists can be considered the forerunners of Nanoscience, which strictly speaking is nothing but the prevalence of surface properties over the bulk properties of a solid.

Surfaces and interfaces are not only relevant in Chemistry, Physics, Catalysis, Materials Science \& Engineering and Nanoscience. Biointerfaces and interfacial phenomena in Biology and Nanomedicine are studied more and more every day. Similarly, surfaces cannot be neglected when targeting applications in many very strategic fields, such as sensors, energy conversion and storage, environmental and food science, and medical devices.

So, surfaces and interfaces represent an intrinsically fascinating topic that cannot be ignored in every interdisciplinary research.

This issue launches a new Open Access journal, Surfaces, that will provide rapid publication of scholarly articles on studies related to surfaces and interfaces. It is our intention to encourage the application of a rigorous Surface Science-based approach to many complex interesting phenomena.

This means:

- publishing important, cutting edge articles and conference proceedings;

- organizing special issues to highlight outstanding research on a specific topic;

- engaging readers like you to submit articles, serve as reviewers, and organize special topical issues of Surfaces;

- making Surfaces a recognized forum for the exchange of the newest advancements of Surface Science.

But, why a further journal on surfaces? All of us are often spammed by several mermaids highlighting new journals. I have been convinced to take the challenge to launch this new journal by two main basic arguments. First, the reliability of the Editorial House, MDPI, who launched several successful Open Access journals; their Editorial Offices are effective enough to fulfil the declared performance. Second, a real interdisciplinary Open Access journal offering the large scope that is reported in the 
following scheme is still lacking. Here, I emphasize the term interdisciplinary, and not multidisciplinary, because one of the goals is break boundaries among different disciplines, harmonizing them into a coordinated and coherent whole.

The scope of Surfaces covers, but is not limited to, the following topics:

- Preparation, functionalization and modification of surfaces and interfaces

- Advanced characterization methods of surfaces and interfaces

- Deposition and growth of thin films

- 2D materials and heterostructures

- $\quad$ Surface and interface physics

- Theoretical and computational studies of surfaces and interfaces

- Surface chemistry and reactivity applied to heterogeneous catalysis, photocatalysis, electrocatalysis, electrified interfaces, semiconductors, surface devices and self-assembled layers

- Coatings and interface engineering

- Adsorption techniques, porous materials and membranes

- Biosurface science, biointerfaces and interfacial phenomena in biology and nanomedicine, biomimetic materials surfaces

- Surface functionalization applied in energy conversion and storage, environmental, food, and medical applications

In addition, while in Biology and Physics there is already a long practice of Open Access publishing, Chemistry has been rather slow in picking up this concept. The main advantages of the Open Access approach are: free access to anyone interested, rapid publication, color figures at no cost (because the journals are published only on the web), and, as it can be freely read by many scientists, including those who do not have access to the expensive subscriber journals, the long term impact is expected to be high. Also, authors can publish their experimental and theoretical results in as much detail as necessary to fully convey the information to others. In our frenetic world, where the speed of transferring information is a solid value, this model has proven to be extremely effective for harnessing new ideas and promoting talents. I hope that Surfaces will be equally authoritative for enhancing research on surfaces across the world.

It is our goal to get the journal off to a strong start in its first year also by publishing a number of special topical issues. As an example, I envision topical issues such as: novel spectroscopic methods for operando investigating surfaces, innovative surface functionalization methods, and photoelectrochemical catalysts. I welcome your suggestions on appropriate guest editors for each of these and on other topics that should be explored.

I look forward to receiving your contributions to Surfaces, and welcome your comments and ideas on how to make this an outstanding journal. I can be reached through the Editorial Office (surfaces@mdpi.com) or through my personal email (gaetano.granozzi@unipd.it).

Conflicts of Interest: The author declare no conflict of interest.

\section{References}

1. Duke, C.B. The birth and evolution of surface science: Child of the union of science and technology. Proc. Natl. Acad. Sci. USA 2003, 100, 3858-3864. [CrossRef] [PubMed]

2. Kambe, K. Theory of Low-Energy Electron Diffraction. Z. Naturf. A 1967, 22, 322-330. [CrossRef]

3. Jona, F. Low-energy electron diffraction (LEED) spectra: Aluminum. IBM J. Res. Dev. 1970, 14, 444-452. [CrossRef]

4. Pendry, J.B. Low Energy Electron Diffraction; Academic Press: Cambridge, MA, USA, 1974.

5. Siegbahn, K. Electron spectroscopy for chemical analysis (E.S.C.A.). In Philosophical Transactions of the Royal Society of London. Series A, Mathematical and Physical Sciences; Royal Society: London, UK, 1970; pp. 33-57. 